\title{
ON MODULAR LATTICES OF ORDER DIMENSION TWO
}

\author{
RUDOLF WILLE
}

\begin{abstract}
In this note, it is shown that a modular lattice has order dimension $\leqq 2$ if and only if it contains no subset isomorphic to one of five described partially ordered sets.
\end{abstract}

The order dimension of a partially ordered set $(S, \leqq)$ is defined as the smallest cardinal number $m$ such that the relation $\leqq$ is the intersection of $m$ (linear) orders on $S$ (Dushnik and Miller [3]). For the order dimension we have the following compactness theorem (Harzheim [4] and also the review of K. A. Baker, MR 43 \#113):

THEOREM $0 . \quad$ Let $(S, \leqq)$ be a partially ordered set, and let $n$ be a natural number. If every finite subset of $S$ has order dimension $\leqq n$, then $(S, \leqq)$ also has order dimension $\leqq n$.

In Baker, Fishburn and Roberts [2], it is shown that for $n \geqq 2$ there is no finite list of partially ordered sets with the property: A partially ordered set $(S, \leqq)$ has order dimension $\leqq n$ if and only if no subset of $S$ is isomorphic to one of the partially ordered sets in the list. Moreover, there does not exist such a list for lattices. The principal result of this note is that for modular lattices and $n=2$ we have a checking list with five finite partially ordered sets. Using Dilworth's theorem, Baker has proved in [1] that a (finite) distributive lattice has order dimension $\leqq n$ if and only if it does not contain the partially ordered set of atoms and coatoms of a boolean lattice with $2^{n+1}$ elements. For the proof of the principal result we need this theorem in the case $n=2$, hence we give a direct proof for this case without using Dilworth's theorem.

If a partial order $\leqq$ on $S$ is the intersection of orders $C_{i}$, we say that $(S, \leqq)$ is represented by the chains $\left(S, C_{i}\right)$; if $S:=\left\{s_{1}, \cdots, s_{n}\right\}$, we describe a chain $(S, C)$ by the sequence $s_{i_{1}} s_{i_{2}} \cdots s_{i_{n}}$ where $\left(s_{i_{j}}, s_{i_{k}}\right) \in C$ if and only if $j \leqq k$.

Received by the editors October 16, 1972 and, in revised form, January 19, 1973.

AMS (MOS) subject classifications (1970). Primary 06A30; Secondary 06A10.

Key words and phrases. Partially ordered set, order dimension, lattice, modular, distributive, finitely generated.

(c) American Mathematical Society 1974 
LEMma 1. The following partially ordered sets have order dimension 3:
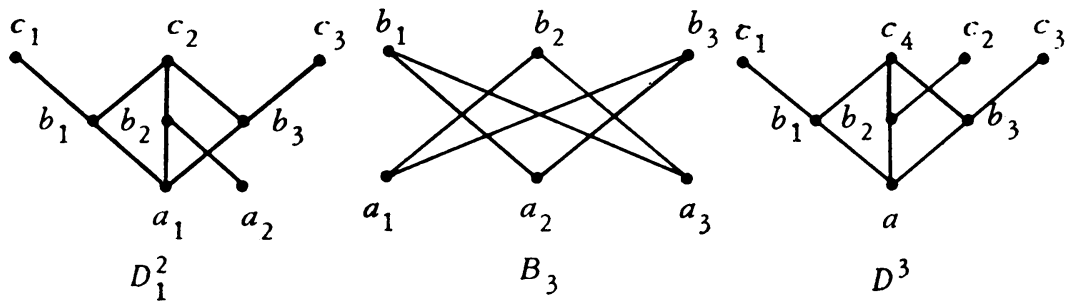

Proof. The following can be easily checked:

$B_{3}$ is represented by

$$
a_{1} a_{2} b_{3} a_{3} b_{1} b_{2}, \quad a_{2} a_{3} b_{1} a_{1} b_{2} b_{3} \text { and } a_{3} a_{1} b_{2} a_{2} b_{3} b_{1},
$$

$D^{3}$ is represented by

$$
a b_{1} c_{1} b_{2} c_{2} b_{3} c_{3} c_{4}, \quad a b_{3} c_{3} b_{2} c_{2} b_{1} c_{1} c_{4} \text { and } a b_{1} b_{2} b_{3} c_{4} c_{1} c_{2} c_{3},
$$

$D_{1}^{2}$ is represented by

$$
a_{1} b_{1} c_{1} b_{3} c_{3} a_{2} b_{2} c_{2}, \quad a_{2} b_{2} a_{1} b_{3} c_{3} b_{1} c_{1} c_{2} \text { and } a_{1} a_{2} b_{1} b_{2} b_{3} c_{2} c_{1} c_{3} .
$$

Now, we have to prove that there is no representation of $B_{3}, D^{3}$ and $D_{1}^{2}$ by any two chains. For $B_{3}$ this follows from the fact that, for every chain of a representation of $B_{3}$, there is at most one $i \in\{1,2,3\}$ with $b_{i} \cdots a_{i}$ in that chain. Suppose $D^{3}$ can be represented by two chains. Then, because of $b_{m} c_{n}$ for $m \neq n \neq 4$, those two chains have to be of the form

and

$$
\cdots b_{i} \cdots c_{i} \cdots b_{j} \cdots c_{j} \cdots b_{k} \cdots c_{k} \cdots
$$

$$
\cdots b_{k} \cdots c_{k} \cdots b_{j} \cdots c_{j} \cdots b_{i} \cdots c_{i} \cdots \quad(\{i, j, k\}=\{1,2,3\}),
$$

which implies that $c_{j} \cdots c_{4}$ is in both chains; this contradicts $c_{j}$ in $D^{3}$. Therefore, $D^{3}$ cannot be represented by two chains. Suppose $D_{1}^{2}$ can be represented by two chains. Then, because of $a_{2}$ those two chains have to be of the form $a_{1} \cdots c_{i} \cdots c_{j} a_{2} \cdots c_{2}$ and

$$
a_{2} a_{1} \cdots c_{2} c_{j} c_{i} \quad(\{i, j\}=\{1,3\}),
$$

which implies that $b_{i} \cdots c_{j}$ is in both chains; this contradicts $b_{i}$ in $D_{1}^{2}$. Therefore, $D_{1}^{2}$ cannot be represented by two chains.

If $D_{3}$ is the dual of $D^{3}$ and $D_{2}^{1}$ the dual of $D_{1}^{2}$, Lemma 1 also shows that $D_{3}$ and $D_{2}^{1}$ have order dimension 3 . For Lemma 2 we need the following notion: An element $c$ of a lattice $L$ with 0 is called a chain element of $L$ if the interval $[0, c]$ is a chain. 
Lemma 2. Let $L$ be a finite distributive lattice having no subset isomorphic to $B_{3}$, and let $c$ be a maximal chain element of $L$. Then $L \backslash[0, c]$ is a sublattice of $L$.

Proof. Obviously, the join of any two elements of $L \backslash[0, c]$ is again in $L \backslash[0, c]$. Suppose there are $a, b \in L \backslash[0, c]$ with $a \wedge b \in[0, c]$. Since $c$ is a maximal chain element of $L$, there exist $a_{1} \leqq a$ and $b_{1} \leqq b$ with $a_{1} \geq c$ and $a_{1} \geq b_{1}$. Obviously, $a_{1} \wedge b_{1} \in[0, c]$. We can assume, without loss of generality, that $a_{1} \wedge c \geqq b_{1} \wedge c$. Let $a_{2}$ and $c_{2}$ be covers of $a_{1} \wedge c$ with $a_{2} \leqq a_{1}$ and $c_{2} \leqq c$, and let $b_{2}$ be a cover of $b_{1} \wedge c$ with $b_{2} \leqq b_{1}$. Then $b_{2} \vee$ $\left(a_{1} \wedge c\right)$ covers $a_{1} \wedge c$; furthermore, $a_{2} \neq b_{2} \vee\left(a_{1} \wedge c\right) \neq c_{2} \neq a_{2}$. By distributivity, it follows that $\left\{a_{2}, b_{2} \vee\left(a_{1} \wedge c\right), c_{2}, b_{2} \vee c_{2}, a_{2} \vee c_{2}, a_{2} \vee b_{2}\right\}$ is a subset of $L$ isomorphic to $B_{3}$. This is a contradiction to our assumption. Therefore, the meet of any two elements of $L \backslash[0, c]$ is again in $L \backslash[0, c]$, too.

THEOREM 3. A distributive lattice has order dimension $\leqq 2$ if and only if it does not contain a subset isomorphic to $B_{3}$.

Proof. By Lemma 1, a distributive lattice of order dimension $\leqq 2$ cannot contain a subset isomorphic to $B_{3}$. Now, let $L$ be a distributive lattice having no subset isomorphic to $B_{3}$. First we prove by induction on the cardinality of $L$ : If $L$ is finite and if $c$ is any chain element of $L$, then $L$ can be represented by two chains $a_{1} a_{2} \cdots a_{n}$ and $b_{1} b_{2} \cdots b_{n}$ such that $[0, c]=\left\{a_{1}, a_{2}, \cdots, a_{i}\right\}$ for some $i \leqq n$. Let $d$ be a maximal chain element of $L$ with $c \leqq d$. By Lemma $2, L \backslash[0, d]$ is a sublattice of $L$, which has a least element $v$. Since $L$ does not contain a subset isomorphic to $B_{3}$, $d \vee v$ is a chain element of $L \backslash[0, d]$. By the induction hypothesis, $L \backslash[0, d]$ can be represented by two chains $a_{1} a_{2} \cdots a_{m}$ and $b_{1} b_{2} \cdots b_{m}$ with $[v, d \vee v]=\left\{a_{1} a_{2}, \cdots, a_{j}\right\}$ for some $j \leqq m$. Now, it can be easily checked that $L$ is represented by the two chains

$$
0 \cdots\left(d \wedge a_{1}\right)\left(d \wedge a_{2}\right) \cdots\left(d \wedge a_{j}\right) a_{1} a_{2} \cdots a_{m}
$$

and

$$
0 \cdots\left(d \wedge a_{1}\right) b_{k_{1}} \cdots b_{k_{2}-1}\left(d \wedge a_{2}\right) b_{k_{2}} \cdots b_{k_{j}-1}\left(d \wedge a_{j}\right) b_{k_{j}} \cdots b_{m}
$$

with $a_{i}=b_{k_{i}}$ for $1 \leqq i \leqq j$. This proves that $L$ has order dimension $\leqq 2$ if $L$ is finite. Now, let $L$ be infinite. Since every finite subset of $L$ generates a finite sublattice of $L$, each finite subset of $L$ has order dimension $\leqq 2$. But then the whole lattice $L$ also has order dimension $\leqq 2$ by Theorem 0 .

Lemma 4. Let $M$ be a modular lattice having no subset isomorphic to $D^{3}$. If $\left\{a, c_{1}, c_{2}, c_{3}, e\right\}$ is a five-element, nondistributive sublattice of $M$ with $a<c_{i}<e$, then $a$ is covered by $c_{i}$ and $e$ covers $c_{i}(i=1,2,3)$. 
Proof. Suppose $a$ is not covered by $c_{i}$ or $e$ does not cover $c_{i}$ for some $i$. Then, by modularity, there is an element $b_{1}$ in $M$ with $a<b_{1}<c_{1}$. Define $b_{2}:=\left(b_{1} \vee c_{3}\right) \wedge c_{2}, \quad b_{3}:=\left(b_{1} \vee c_{2}\right) \wedge c_{3}$ and $c_{4}:=\left(b_{1} \vee c_{2}\right) \wedge\left(b_{1} \vee c_{3}\right)$. Since $a<b_{i}<c_{i}$ and $c_{i} \wedge c_{4}=b_{i}$ for $1 \leqq i \leqq 3$, the subset $\left\{a, b_{1}, b_{2}, b_{3}, c_{1}, c_{2}, c_{3}, c_{4}\right\}$ is isomorphic to $D^{3}$.

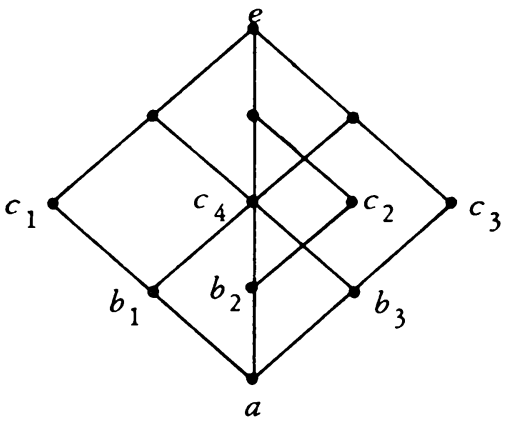

An element $a$ of a lattice $L$ is said to be irreducible if $b_{1}, c_{1}<a$ implies $b_{1} \vee c_{1}<a$ and if $a<b_{2}, c_{2}$ implies $a<b_{2} \wedge c_{2}$. Thus, irreducible elements are exactly those elements which can be deleted from a sublattice with the end result that a sublattice remains. Especially, $\mathfrak{N}(L):=\{b \in L \mid b$ is not irreducible is a sublattice of $L$.

THEOREM 5. For a modular lattice $M$ the following conditions are equivalent:

(i) $M$ has order dimension $\leqq 2$.

(ii) $\mathfrak{N}(M)$ is distributive and has order dimension $\leqq 2$.

(iii) $M$ does not contain a subset isomorphic to $B_{3}, D^{3}, D_{3}, D_{1}^{2}$ or $D_{2}^{1}$.

Proof. Since the order dimension of any subset of $M$ cannot exceed the order dimension of $M$, (i) $\Rightarrow$ (iii) is a consequence of Lemma 1 .

(iii) $\Rightarrow$ (ii): Let $\left\{a, b_{1}, b_{2}, b_{3}, c\right\}$ a five-element, nondistributive sublattice of $M$. By Lemma $4, a$ is covered by $b_{i}$ and $c$ covers $b_{i}(i=1,2,3)$. Since no subset of $M$ is isomorphic to $D^{3}, D_{3}, D_{1}^{2}$ or $D_{2}^{1}$, it follows that at least one of the $b_{i}$ 's has to be irreducible. Therefore, $\left\{a, b_{1}, b_{2}, b_{3}, c\right\}$ is not contained in $\mathfrak{N}(M)$. Thus $\mathfrak{N}(M)$ is distributive and has order dimension $\leqq 2$ by Theorem 3 .

(ii) $\Rightarrow$ (i): Since $\mathfrak{N}(M)$ is distributive, every five-element, nondistributive sublattice $M_{3}$ of $M$ must contain an irreducible element $c$, and we get by the same construction as in the proof of Lemma 4 that $c$ has a cover $c^{-}$ and a subcover $c_{-}$in $M$ with $c^{-}, c_{-} \in M_{3}$. Let $S$ be a sublattice of $M$ generated by a finite subset $F$, and let $I$ be the set of all irreducible elements of $F$ contained in a five-element, nondistributive sublattice of $M$. We define $I^{-}:=\{c \mid c \in I\}$ and $I_{-}:=\left\{c_{-} \mid c \in I\right\}$. Since $(F \mid I) \cup I^{-} \cup I_{-}$is 
contained in the distributive sublattice $\mathfrak{N}(L)$, the finite set $(F \backslash I) \cup \Gamma \cup I$ generates a finite sublattice $T$ of $M$. Furthermore, we have $a \vee c=a \vee c=$ and $b \wedge c=b \wedge c_{-}$for $a, b \in M$ and $c \in I$ if $a \vee c \neq c \neq b \wedge c$. Hence $S \subseteq T \cup I$, and therefore $\bar{S}$ is finite. Thus, every finitely generated sublattice of $M$ is finite. Therefore, by Theorem 0 , we can assume that $M$ is finite. Let $D$ be a maximal distributive sublattice of $M$ with $\mathfrak{N}(M) \subseteq D$. By Theorem 3 and (ii), $D$ has order dimension $\leqq 2$. Now, we prove for every subset $E \supseteq D$ by induction on the cardinality of $E$ that $E$ has order dimension $\leqq 2$. Suppose we have already seen that $E$ can be represented by two chains $a_{1} a_{2} \cdots a_{r}$ and $b_{1} b_{2} \cdots b_{r}$. If there is a $c \in M$ with $c \notin E, c$ is an irreducible element contained in a five-element, nondistributive sublattice. Since $D$ is a maximal distributive sublattice containing $\mathfrak{N}(L)$ and since the length of the interval $\left[c_{-}, c^{-}\right]$is two, there are exactly four elements $c_{-}, b, d, c^{-}$ in the intersection $D \cap\left[c_{-}, c^{-}\right]$. There is, without loss of generality, $i<k$ with $a_{i}=b$ and $a_{k}=d$. Let $j$ be the smallest number with $i<j$ and $a_{i}$ a $a_{j}$. Furthermore, there is $l<n$ with $b_{l}=d$ and $b_{n}=b$. Let $m$ be the greatest number with $m<n$ and $b_{m}$

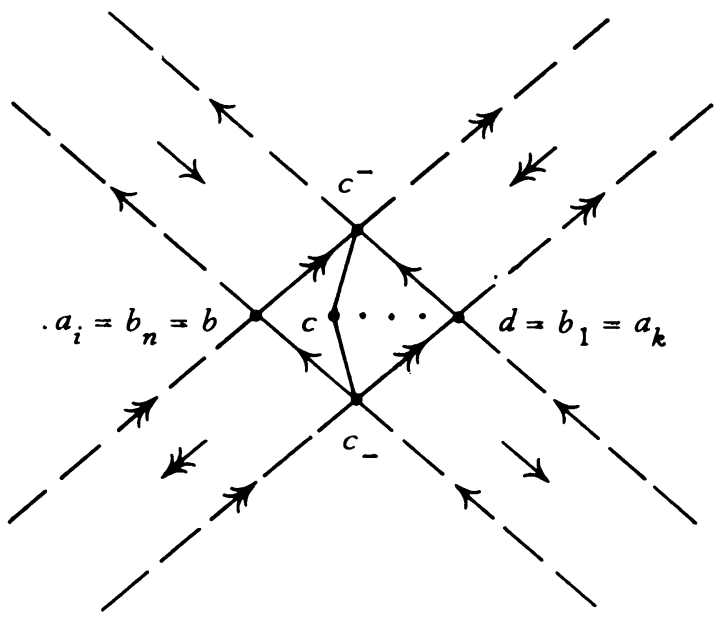

Now, we assert that $E \cup\{c\}$ is represented by $a_{1} \cdots a_{j-1} c a_{j} \cdots a_{r}$ and $b_{1} \cdots b_{m} c b_{m+1} \cdots b_{r}$. Since $x<c$ and $c<y$ implies $x \leqq c_{-}$and $c-\leqq y$, the two orders extend the partial order on $E \cup\{c\}$. Next we have to prove that $a_{p}=b_{q}$ with $j \leqq p$ and $m<q$ implies $c<a_{p}=b_{q}\left(c^{-} \leqq a_{p}=b_{q}\right)$. Suppose $c^{-} a_{p}=b_{q}$. Then we have $a_{i} a_{p}$ or $b_{l} b_{q}$. If $a_{i} a_{p}$, by $i<p$, it follows $q<n$. Because of $m<q$, we get $a_{p}=b_{q} \leqq b_{n}=a_{i}$ which contradicts $i<p$. If $a_{i} \leqq a_{p}$, we must have $b_{l} \leqq b_{q}$. This forces $p<k$ because of $l<q$. Since $a_{i} \leqq a_{p}$, we have $b_{n} \leqq b_{q}$ and hence $n \leqq q$. If we let $c^{-}=b_{s}=a_{t}$ and $a_{j}=b_{w}$, then $k<t, n<s$, and $w<n$. Thus $w<q, w<s$, and $j<t$ because $p<k$. 
Hence $c^{-} a_{p}, a_{i} \leqq a_{p}$ and $a_{i} \leq a_{j}$ implies $a_{j} \leqq a_{p}$ and $a_{j} \leqq c^{-}$. Since $c^{-}$ covers $a_{i}$, we get $a_{j} \leqq a_{p} \wedge c^{-}=a_{i}$ which contradicts $i<j$. Thus, $c^{-} \leqq a_{p}=b_{q}$ is proved. Dually, it follows that $a_{q}=b_{p}$ with $q<j$ and $p \leqq m$ implies $a_{q}=b_{p}<$ $c\left(a_{q}=b_{p} \leqq c_{\ldots}\right)$. This finishes the proof that $E \cup\{c\}$ is represented by $a_{1} \cdots$ $a_{j-1} c a_{j} \cdots a_{r}$ and $b_{1} \cdots b_{m} c b_{m+1} \cdots b_{r}$. Therefore, $M$ has order dimension $\leqq 2$.

COROllary 6. Every finitely generated modular lattice of order dimension $\leqq 2$ is finite.

Problem. For which natural numbers $n \geqq 3$ does there exist a finite list of partially ordered sets $\left(S_{i}, \leqq_{i}\right)$ such that a modular lattice has order dimension $\leqq n$ if and only if it contains no subset isomorphic to some of the $\left(S_{i}, \leqq_{i}\right)$.

\section{REFERENCES}

1. K. A. Baker, Dimension, join-independence, and breadth in partially ordered sets, Honors Thesis, Harvard University, Cambridge, Mass., 1961.

2. K. A. Baker, P. C. Fishburn and F. S. Roberts, Partial orders of dimension 2, interval orders, and interval graphs, Mimeographed, Rand Corporation, 1970.

3. B. Dushnik and E. W. Miller, Partially ordered sets, Amer. J. Math. 63 (1941), 600-610. MR 3, 73.

4. E. Harzheim, Ein Endlichkeitssatz über die Dimension teilweise geordneter Mengen, Math. Nachr. 46 (1970), 183-188. MR 43 \#113.

Fachbereich Mathematik, Technische Hochschulle Darmstadt, Federal Republic of Germany 